\title{
Wide-Band Generation of Picosecond Pulse Using Fiber Optical Parametric Amplifier and Oscillator
}

\author{
Yue Zhou, Bill P. P. Kuo, Kim K. Y. Cheung, Sigang Yang, P. C. Chui, and Kenneth K. Y. Wong, Member, IEEE
}

\begin{abstract}
We first demonstrate a wideband generation of picosecond pulse using fiber optical parametric amplifier (FOPA). High quality pulse is generated at $85 \mathrm{~nm}$ away from the pump with pulsewidth narrower than that of the pump. We then explore fiber optical parametric oscillator (FOPO) configuration which has the advantage of eliminating the need of seeding laser, leading to a potentially wider wavelength tunability and flexibility. The tuning range of the FOPO is from $1511 \mathrm{~nm}$ to $1541 \mathrm{~nm}$ and from $1583 \mathrm{~nm}$ to $1613 \mathrm{~nm}$, which is as wide as $60 \mathrm{~nm}$, with wavelength span of over $100 \mathrm{~nm}$. Nearly-transform-limited sub-picosecond pulses are generated by this technique.
\end{abstract}

Index Terms-Optical parametric amplifier, optical parametric oscillator, optical pulse generation.

\section{INTRODUCTION}

$\mathbf{P}$ ICOSECOND optical pulse sources have been playing an important role in the study of ultrafast processes and there is a growing demand of short pulse generation in non-conventional wavelengths in ultrafast optical communications. However, high-quality picosecond laser is generally not available at some non-conventional wavelengths due to the lack of efficient gain medium. Fiber optical parametric amplifier [1], offers ultra-fast response, wide-gain bandwidth [2], high gain [3] and large wavelength detune from the pump [2], allows efficient short pulse generation at non-conventional wavelengths via idler generation.

Previous efforts include utilizing conventional parametric gain to generate picosecond pulse over C/L-band [4]. In our previous work, we also proposed and demonstrated the generation of picosecond pulse at large wavelength detune $(\sim 100 \mathrm{~nm})$ from the pump using higher-order-dispersion-mediated phase matching in highly-nonlinear fiber (HNLF) [5].

The availability of continuous-wave (CW) lasers at certain wavelength $(\sim 1640 \mathrm{~nm})$ or even longer wavelength limits the

Manuscript received March 20, 2009; revised July 07, 2009. Current version published October 21, 2009. This work was supported in part by Grants from the Research Grants Council of the Hong Kong Special Administrative Region, China (Project No. HKU 7172/07E and HKU 7179/08E).

Y. Zhou, K. K. Y. Cheung, S. Yang, P. C. Chui, K. K. Y. Wong are with the Photonic Systems Research Laboratory, Department of Electrical and Electronic Engineering, University of Hong Kong, Hong Kong (e-mail: yzhou@eee. hku.hk; cheungky@eee.hku.hk; sgyang@eee.hku.hk; pcchui@eee.hku.hk; kywong@eee.hku.hk).

Bill P. P. Kuo is with the Department of Electrical and Computer Engineering, University of California at San Diego, La Jolla, CA 92093 USA (e-mail: p2kuo@ucsd.edu).

Color versions of one or more of the figures in this paper are available online at http://ieeexplore.ieee.org.

Digital Object Identifier 10.1109/JQE.2009.2030691 tunable range of this kind of picosecond source. Therefore, it is important to explore its corresponding oscillator configuration, which does not require any seeding light.

Conventionally, optical parametric oscillator (OPO) based on $\chi^{(2)}$ nonlinear effect of crystals is utilized for parametric oscillation [6]. This technique has a wide range of applications in optical research and development. However, this approach requires a dedicated alignment for optimal operation. Fiber optical parametric oscillators (FOPO), which based on $\chi^{(3)}$ nonlinear effect of optical fiber, eliminate the need for alignment and allow further integration with fiber components [7]. Using FOPO configuration, CW [8]-[10] and nanosecond [11] pulse generators have been demonstrated with wide wavelength tuning range. In the visible regime, widely-tunable picosecond FOPOs based on microstructure fiber (MF) have been demonstrated [12]-[15]. However, most telecommunication applications require sources in the spectral region near $1550 \mathrm{~nm}$. Some other MF based FOPO [16], DSF based FOPO [17] and HNLF based FOPOs [18], [19] were designed to operated in this regime, but usually with limited tuning range $(\sim 40 \mathrm{~nm})$. In our previous work, picosecond FOPOs were demonstrated with tuning range of $250 \mathrm{~nm}$ around $1550 \mathrm{~nm}$ by tuning the pump wavelength [20] and $150 \mathrm{~nm}$ around 1550 $\mathrm{nm}$ by changing the cavity length [21]. The outputs were 10-ps pulse trains with linewidths of $2 \mathrm{~nm}$. However, the fairly large time-bandwidth products (TBP) of the pulses indicated that they were far from transform-limited. Since transform-limited pulse generation is crucial to the success of long distance and high-bit-rate transmissions, building a widely-tunable FOPO with nearly-transform-limited pulse around $1550 \mathrm{~nm}$ is highly desirable.

This paper is organized as follows. In Section II, the principle of FOPA and FOPO is investigated. In Section III, the previous picosecond pulse generation method using FOPA [5] is presented and the influence of the walk-off effect on signal pulsewidth is discussed. In Section IV, we then demonstrate a widely-tunable picosecond FOPO based on parametric gain by the same spool of highly-nonlinear dispersion-shifted fiber (HNL-DSF) but in a ring cavity configuration. This ring cavity is synchronously pumped with a picosecond mode-locked fiber laser (MLFL). Nearly-transform-limited sub-picosecond pulse is generated. The tuning range is as wide as $60 \mathrm{~nm}$ around $1550 \mathrm{~nm}$, with wavelength span of over $100 \mathrm{~nm}$. In Section V, we conclude our work and illustrate the future applications of our scheme, such as ultrafast communication in non-conventional wavelength bands. 


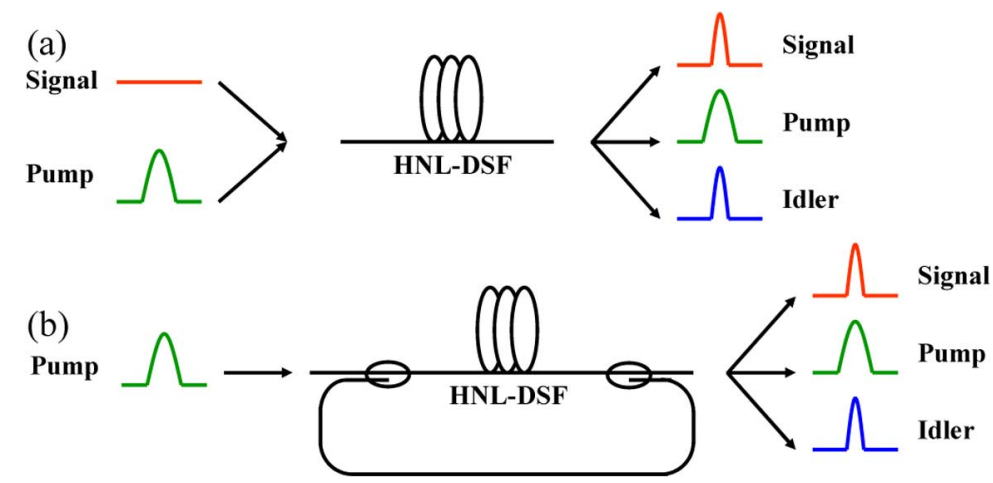

Fig. 1. The principle of pulse generation in FOPA (a) and FOPO (b).

\section{PRINCIPLE}

Fig. 1(a) shows the schematic diagram illustrating the principle of pulse generation in FOPA.

When the pulsed pump propagates with a CW signal through a nonlinear gain medium (e.g., HNL-DSF) for parametric amplification, the signal is amplified and a new wavelength component called idler is generated. When the same spool of HNL-DSF is deployed as the gain medium inside a cavity, such as Fig. 1(b), only one pump is required as the input to the system. The parametric fluorescence will be amplified and fed back to the HNL-DSF as a seed. After multiple trips there will be significant signal and idler components at the output.

The signal and idler waveforms follow the pulse shape of the pulsed-pump as the amplification in FOPA depends on the instantaneous pump power. The pulsewidth of the generated signal and idler pulse is narrower than that of the pump pulse due to pulse compression effect inherited from exponential gain of FOPA [4].

Phase-matching technique is required in order to generate signal and idler with substantial power. The phase matching condition is

$$
\Delta \beta+2 \gamma P=0
$$

where $\gamma$ is the fiber nonlinear coefficient, $P$ is the incident pump power. $\Delta \beta$ is the total phase mismatch, which can be approximated by the following equation [2]:

$$
\Delta \beta=\beta^{(2)}(\Delta \omega)^{2}+\beta^{(4)}(\Delta \omega)^{4} / 12
$$

where $\Delta \omega$ is the frequency detune of signal (and therefore idler) from the pump, $\beta^{(\mathrm{m})}$ is the mth order derivative of the propagation constant at the pump wavelength.

The shape of the gain spectrum is determined by the fiber dispersion profile. When $\beta^{(2)}<0$ and $\beta^{(4)}<0$, it will operate as a conventional FOPA, which has a continuous gain spectrum around the pump. One the other hand, when $\beta^{(2)}>0$ and $\beta^{(4)}<0$, i.e., the pump is in the normal dispersion regime, it is possible to generate idler and signal with frequency detune larger than the bandwidth of conventional FOPA.

\section{Pulse Generation Using FOPA}

The experimental setup of wideband picosecond pulse generator is shown in Fig. 2. The pulsed pump can be generated by the sources shown in either inset (a) or inset (b) in Fig. 2 for different purposes (explained later). It is then amplified by EDFA1 and EDFA2, and filtered by TBPF1 and TBPF2 to produce a high-power, low-noise pump. The amplified pump is monitored through a 10/90 coupler and combined with a CW signal light from DFB laser. The combined light waves then pass through a circulator, and the reflected power is observed using a power meter. Since the linewidth of the pump pulse is larger than the bandwidth of the stimulated Brillouin scattering (SBS) gain, the SBS is low and therefore the phase modulator used in [10] is not required to suppress the SBS. Afterwards, the signal and the pump are launched into a spool of 50-m HNL-DSF with nonlinear coefficient $14 \mathrm{~W}^{-1} \mathrm{~km}^{-1}$, zero-dispersion wavelength (ZDW) $1554.7 \mathrm{~nm}$ and dispersion slope $0.035 \mathrm{ps} / \mathrm{nm}^{2} / \mathrm{km}$ for parametric process. The output spectrum is monitored at an optical spectrum analyzer (OSA) through a 1/99 coupler, the idler in S-band is selected through two WDM combiners and the waveform is observed using a digital communication analyzer (DCA). The receiver module used in the DCA is Agilent 86116A, which has an operating wavelength of 1000-1600 nm, an optical bandwidth of $53 \mathrm{GHz}$ and an electrical bandwidth of $63 \mathrm{GHz}$.

The OPA gain spectra are examined using the pulse source shown in inset (a) of Fig. 2. The pulsed pump is generated by intensity-modulating a CW DFB laser with narrow electrical pulse of $0.2 \%$ duty ratio and $200 \mathrm{kHz}$ repetition rate. Thanks to slow gain dynamics of EDFA, pulse with high peak power is obtained with this low-duty-ratio input pulse. The pulsed pump is amplified to peak power of $20 \mathrm{~W}$ as measured after TBPF2 and the corresponding spontaneous emission spectra are shown in Fig. 3.

Fig. 3 shows the spontaneous emission spectra from FOPA when the pump pulsewidth is $10 \mathrm{~ns}$ and pump wavelength is tuned from $1558 \mathrm{~nm}$ to $1542 \mathrm{~nm}$. It is observed that the FOPA-generated spontaneous emission spectra, which estimate the gain, evolve from a continuous band for long pump wavelength to pairs of narrow discrete bands for pump wavelength below ZDW. The gain peak shifts gradually away from the pump when the pump moves further into normal dispersion regime, where the maximum separation of $98.8 \mathrm{~nm}$ between pump and gain peak is attained when the pump is at $1544 \mathrm{~nm}$. Even though the exact reason for the limitation of the maximum separation is not yet clear, fluctuations of the ZDWs of the 


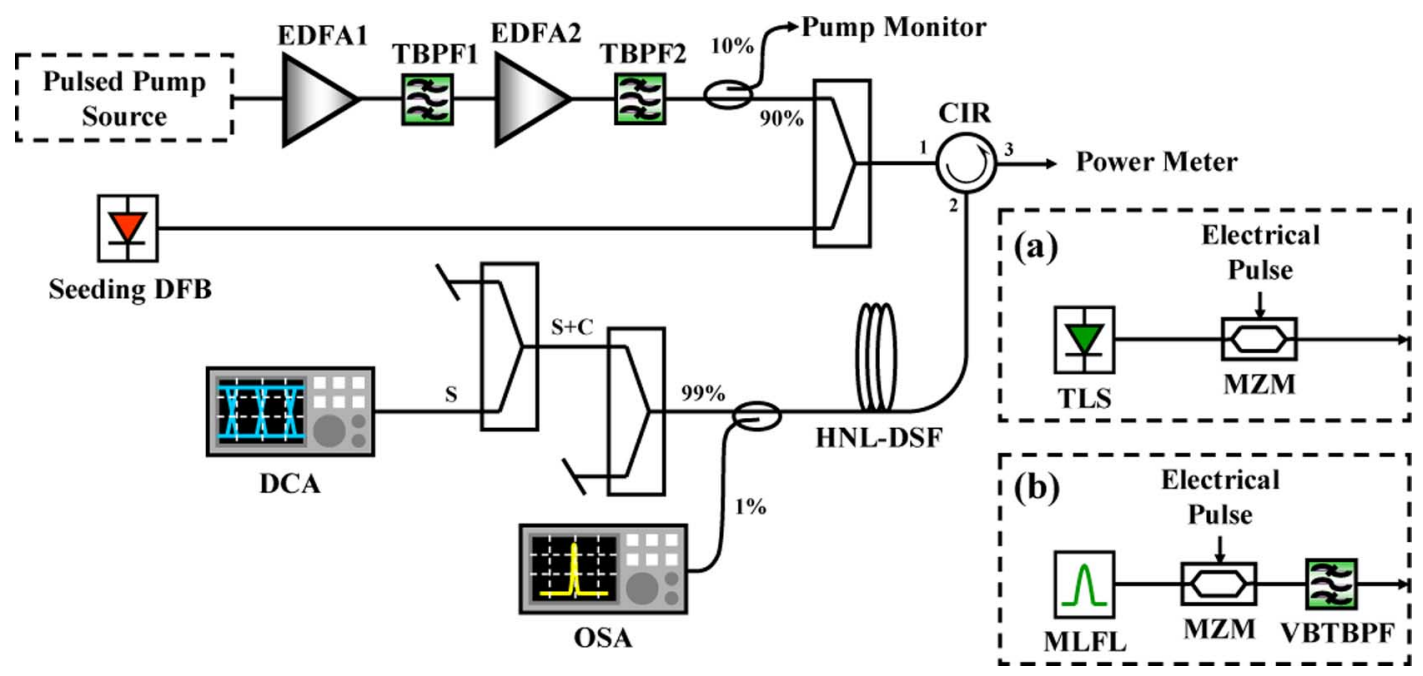

Fig. 2. Experimental setup of OPA-based pulse generator. Insets show the configuration of pulsed pump source. EDFA: erbium-doped fiber amplifier, TBPF: tunable bandpass filter, MZM: Mach-Zehnder modulator, DFB: Distributed Feedback, CIR: circulator.

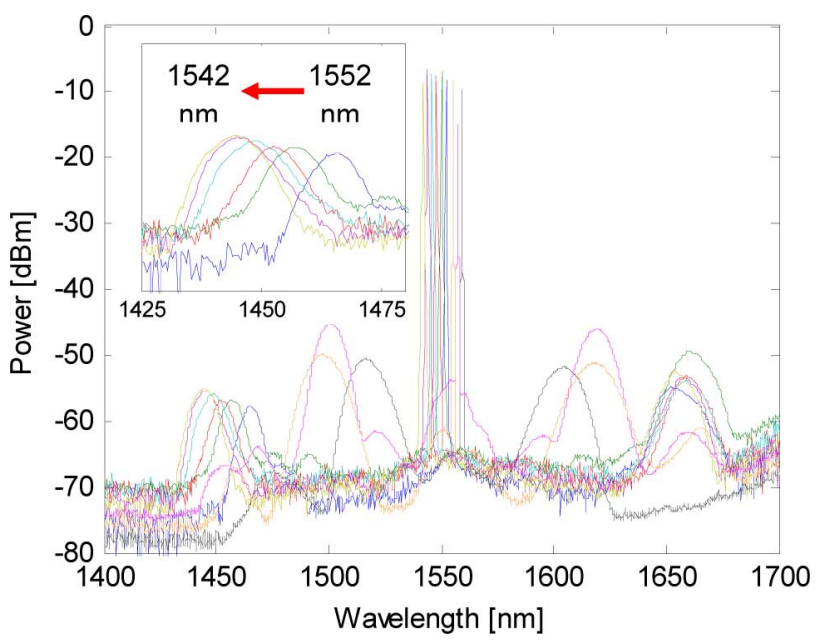

Fig. 3. Spontaneous emission spectra of the narrowband OPA. Inset shows the expanded view of narrowband gain spectra in S-band.

fiber have been investigated systematically and shown to be a detrimental factor for the parametric gain and efficiency [22], [23]. However, researchers reported that the bandwidth limitation is not a result of dispersion fluctuations along the fiber by comparing the tuning range of FOPA with different lengths of fibers [11], [24]. Further research is required to understand the exact reason of the limitation of the maximum separation.

Note that the previous pulsed pump (inset (a) of Fig. 2) is deployed to identify various gain spectra peak. To generate picosecond pulse, the nano-second pulse source is replaced by the picosecond pulse source shown in inset (b) of Fig. 2. The pump is generated by a picosecond mode-locked fiber laser (MLFL) which generated short pulse with repetition rate of $10 \mathrm{GHz}$, pulsewidth of $10 \mathrm{ps}$ and center wavelength of $1545 \mathrm{~nm}$. The output from pulsed laser is intensity-modulated and filtered by a variable-bandwidth tunable bandpass filter (VBTBPF) to produce pump pulse with duty ratio of $0.17 \%$ and pulsewidth of 11.2 ps.
Fig. 4 shows the spectra at HNL-DSF input and output. From the spectra, it can be observed that the pump, signal and idler suffer from spectral broadening due to self-pahse modulation (SPM) of the pump and cross-phase modulation (XPM) of the signal and idler due to the pump. A more accurate value of the FOPA gain can be obtained when the resolution of the spectra is reduced from $1 \mathrm{~nm}$ to $20 \mathrm{~nm}$ by running average. From the averaged spectra, the signal attains average gain of $6.3 \mathrm{~dB}$. Suppose the duty ratio of the pump pulse is $d$, and the power of the input $\mathrm{CW}$ signal is $P_{\text {in }}$. After parametric amplification by a pulsed pump, only a portion of the signal will be amplified, from peak power $d \times P_{\text {in }}$ to $P_{p}$, and the total power of the amplified signal will be $(1-d) \times P_{\text {in }}+P_{p}$. The average gain $G_{a}$ can be calculated using

$$
G_{a}=10 \log \left(\frac{P_{p}+(1-d) \times P_{\text {in }}}{P_{\text {in }}}\right)
$$

and the peak gain $G_{p}$ can be calculated using

$$
G_{p}=10 \log \left(\frac{P_{p}}{d \times P_{\text {in }}}\right) .
$$

Thus, the relationship between the peak gain and the average gain is

$$
G_{p}=10 \log \left(10^{\frac{G_{a}}{10}} \times \frac{1}{d}-\frac{1-d}{d}\right) .
$$

The peak gain is calculated to be $32.8 \mathrm{~dB}$. Fig. 5 shows the waveforms of input pump pulse and output idler pulse. As can be observed from the waveforms, the pulsewidth of idler pulse is shorter than that of the pump pulse while having signal-to-noise ratio (SNR) of $22.5 \mathrm{~dB}$. The pulse narrowing effect of the idler pulse is contributed by the exponential gain nature of FOPA. The actual idler pulsewidth is believed to be shorter than the measured value as the high amplitude ringing at pulse trailing edge suggests the measurement is limited by the bandwidth of the receiver in DCA.

However, the pulse compression ratio is less than the theoretical value of 4 to 5 [4]. This is primarily caused by significant 

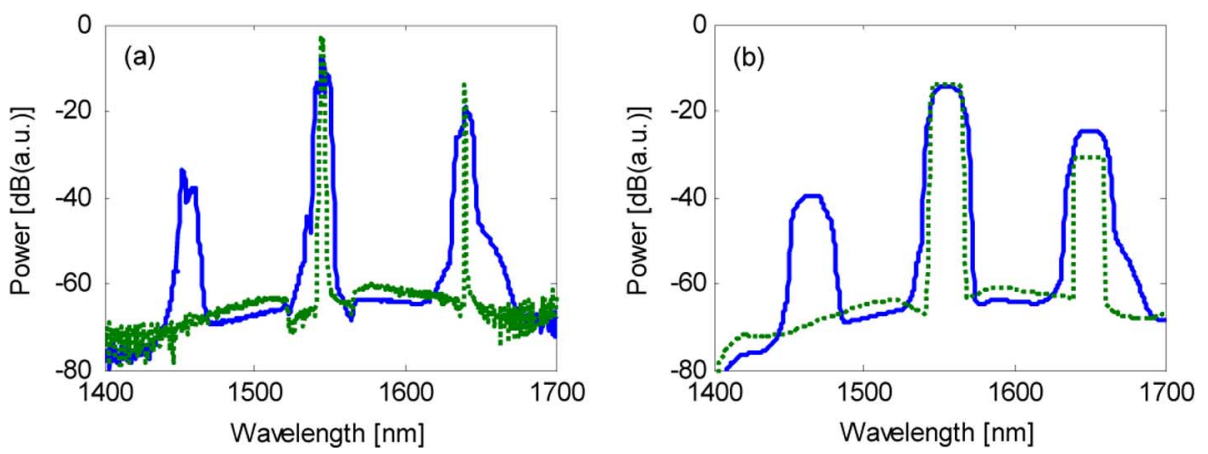

Fig. 4. (a) Optical spectra (resolution $1 \mathrm{~nm}$ ) measured at HNLF input (dotted) and output (solid); (b) Averaged spectra (resolution $20 \mathrm{~nm}$ ).

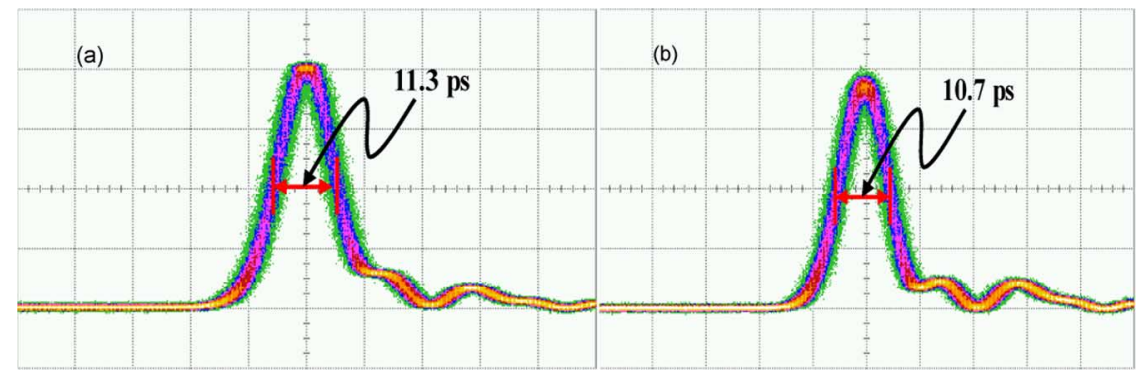

Fig. 5. (a) Output pulse from the pump source and (b) idler pulse observed at HNL-DSF output. Horizontal scale is $10 \mathrm{ps} / \mathrm{div}$.

walk-off between the generated idler and the pump, which can be explained as follows.

To understand the effect of pulse walk-off in OPA, one may model the fiber as a large number of repeating units, where each unit consists of a nonlinear section responsible for OPA effect and a linear section for chromatic dispersion, as shown in Fig. 6. Note that it is similar to the concept in split-step Fourier method (SSFM) [25]. In the first nonlinear section, the pump pulse amplifies part of the signal and generates idler pulse. When the pump, signal and idler go through the linear section, walk-off occurs as consequence of their difference in group velocities. In the normal dispersion regime $(<1554.7 \mathrm{~nm})$, longer wavelength components travels faster than the shorter wavelength componets [25], so the pump travels faster than the idler; on the contrary, in the anomalous-dispersion regime, shorter wavelength travels faster than the longer wavelength, so the pump also travels faster than the signal. Since the pump travels faster than the other waves, the pump pulse will displace from the signal and idler pulse. As a result, in the second nonlinear section, the pump pulse interacts with a different portion of the signal and idler wave which in effect generates a broader pulse. As the waves propagates along the fiber, the walk-off phenomenon aggregate and results in generating a broader pulse with less efficiency.

The walk-off, which is the time separation between signal (idler) and the pump after a single path through the fiber can be calculated approximately using the following equation [25]:

$$
\Delta T=\left|t\left(\lambda_{S}\right)-t\left(\lambda_{P}\right)\right| \approx L\left|D\left(\lambda_{c}\right)\right|\left|\lambda_{S}-\lambda_{P}\right|
$$

where $\lambda_{\mathrm{S}}$ is the signal wavelength, $\lambda_{\mathrm{P}}$ is the pump wavelength, $\lambda_{\mathrm{c}}=\left(\lambda_{\mathrm{S}}+\lambda_{\mathrm{P}}\right) / 2, t(\lambda)$ is the propagation time of the wave-

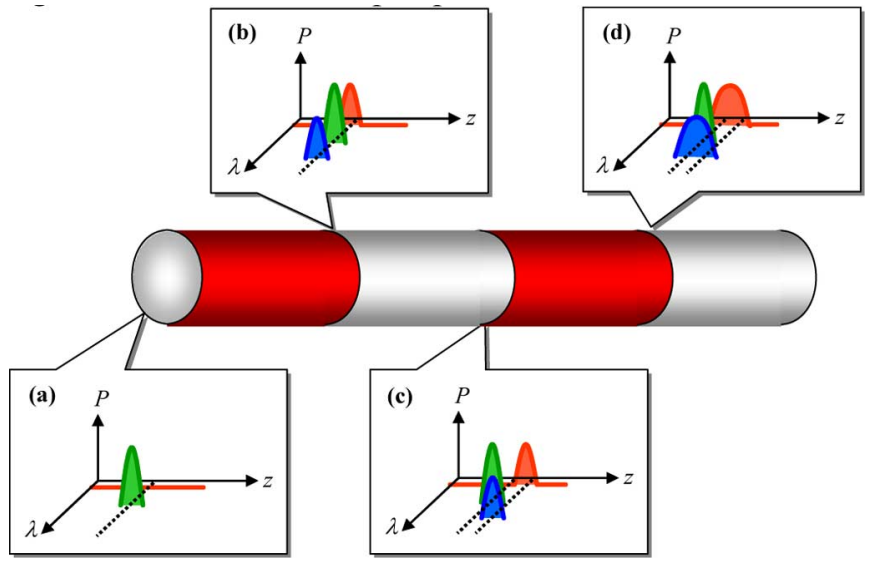

Fig. 6. Illustration of pulse walk-off effect in pulsed pump OPA. (a) Input CW signal and pulsed pump; (b) signal, pump and idler after 1st nonlinear section; (c) output at after dispersive section; (d) output of 2nd nonlinear section.

length $\lambda$ after a single path through the fiber, $L$ is the fiber length, $D(\lambda)$ is the dispersion at wavelength $\lambda$, which can be calculated using

$$
D(\lambda) \approx D^{\prime}\left(\lambda-\lambda_{0}\right)
$$

where $D^{\prime}$ is the dispersion slope of the fiber, $\lambda_{0}$ is the ZDW of the fiber.

For $\lambda_{\mathrm{S}}=1460 \mathrm{~nm}$ and $\lambda_{\mathrm{P}}=1545 \mathrm{~nm}, \lambda_{\mathrm{c}}$ will be $1502.5 \mathrm{~nm}$. Then if we use (6) and (7) by considering the following fiber parameters: a ZDW of $1554.7 \mathrm{~nm}$ and a dispersion slope of $0.035 \mathrm{ps} / \mathrm{nm}^{2} / \mathrm{km}$, the fiber dispersion at $1502.5 \mathrm{~nm}$ is calculated to be $-1.8 \mathrm{ps} / \mathrm{nm} / \mathrm{km}$, and then the walk-off $(\Delta T)$ is calculated to be $7.65 \mathrm{ps}$, which is comparable to the pump 


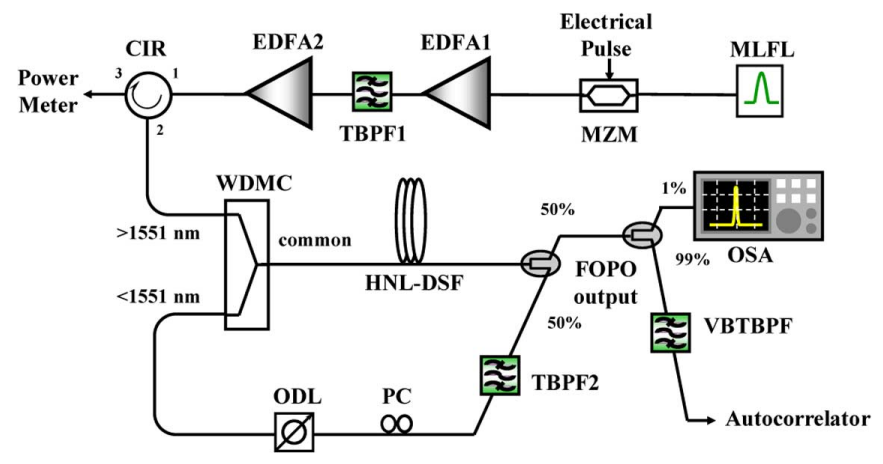

Fig. 7. Experimental setup of FOPO-based pulse generator. ODL: optical delay line; PC: polarization controller.

pulsewidth. Therefore, if it is required to generate narrower picosecond pulses, the walk-off between the pump and the signal should be reduced.

\section{Pulse Generation Using FOPO}

While the previous setup is able to generate pulses at large frequency detune from the pump using FOPA configuration, a seeding $\mathrm{CW}$ laser is required. However, the availability of $\mathrm{CW}$ lasers at certain wavelength potentially limits the tuning range of previous setup, we therefore use FOPO configuration which has a potentially wider tunability. Furthermore, in order to generate nearly-transform-limited sub-picosecond pulses, the walk-off between the signal and the pump would become a critical factor in limiting the signal pulsewidth as discussed in the previous section. Therefore, it is required to compromise the frequency detune to achieve a narrower pulsewidth for the same spool of HNL-DSF. The experimental setup of widely-tunable sub-picosecond pulse generator is shown in Fig. 7. The pump is generated by a MLFL which generated short pulse with repetition rate of 10-GHz, pulsewidth of 2-ps. The output from pulsed laser is intensity-modulated by a $156.2-\mathrm{MHz}$ electrical pulse with duty ratio of $1 / 64$ to increase the peak power of the pump. It is then amplified by EDFA1 and EDFA2, and filtered by TBPF1 with a 1-nm bandwidth to produce a high-power, low-noise pump. The pump is broadened to $3.5 \mathrm{ps}$ by the TBPF1. Afterwards, it pass through a circulator and the reflected power by SBS is monitored using a power meter. The average power of the pump is measured to be $60 \mathrm{~mW}$ after the circulator. Since the amplified spontaneous emission (ASE) noise in the pump is also amplified by the EDFAs, the peak power of the pump is measured to be only $5 \mathrm{~W}$ using a DCA. Afterwards, it is coupled into the cavity for parametric amplification through the wavelength-division multiplexing coupler (WDMC), which has a cutoff wavelength of $1551 \mathrm{~nm}$.

The same spool of HNL-DSF is deployed as the gain medium inside the cavity. A 50/50 coupler in the cavity provided 50\% feedback and $50 \%$ output. The feedback branch is filtered by the TBPF2 with a bandwidth of $1 \mathrm{~nm}$, so that only the idler (antiStokes wave) returns to the HNL-DSF through WDMC. Tuning is achieved by adjusting the center wavelength of TBPF2, from $1500 \mathrm{~nm}$ to $1550 \mathrm{~nm}$. As a result, the FOPO is only singly resonant with the idler. The PC inside the cavity is used to align the state of polarization (SOP) of the idler with that of the pump,

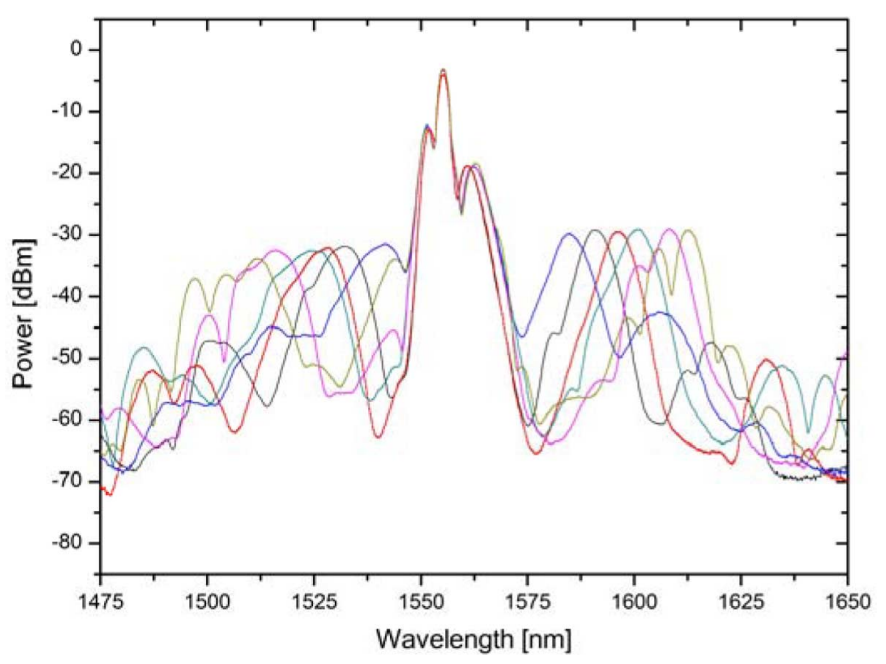

Fig. 8. Optical spectra measured at FOPO output when pump was at $1555 \mathrm{~nm}$ by tuning the intra-cavity TBPF2 as in Fig. 7.

while the ODL in the cavity is used to adjust the cavity length thus the round trip time of the idler matches the pump repetition rate, so as to synchronize the idler with the pump.

The round trip loss of the cavity is measured to be $8.3 \mathrm{~dB}$. The gain is calculated to be $\sim 24 \mathrm{~dB}$ in the case of perfect phase matching [11]:

$$
G_{\mathrm{dB}} \approx 10 \log _{10}\left[\frac{1}{4} \exp (2 \gamma P L)\right]
$$

where $L$ is the fiber length, $P$ is the peak power of the pump, $\gamma$ is the nonlinear coefficient. The actual gain is perceived to be lower than this value, since the ZDW fluctuations and random birefringence along the HNL-DSF would lower the gain [2]. However, the gain can still exceed the cavity loss, such that stable oscillations can be achieved.

The FOPO output spectrum is monitored by an OSA through a 1/99 coupler. A VBTBPF with a 4-nm bandwidth is used to filter out the desired signal or idler. The pulsewidth of the signal (idler) is measured using an autocorrelator.

Fig. 8 shows the optical spectra measured at the FOPO output port. The pump wavelength is fixed at $1555 \mathrm{~nm}$ and its spectrum is broadened due to SPM. Wavelength tuning can be achieved by tuning the center wavelength of the TBPF2 inside the cavity, and adjusting the ODL to compensate the GVD-induced cavity length change [21]. The pump power is slightly adjusted to maintain almost the same peak for all spectra. The achievable output tuning range is from $1511.5 \mathrm{~nm}$ to $1541.7 \mathrm{~nm}$ and from $1583.1 \mathrm{~nm}$ to $1613.2 \mathrm{~nm}$, which is as wide as $60 \mathrm{~nm}$, with wavelength span of over $100 \mathrm{~nm}$. The smaller peaks occurred are higher order four-wave mixing (FWM) components. Further tuning is limited by 1) the walk-off between the pump and the signal, which increases with their wavelength separation and thereby decreases their interaction length 2) the gain region of the FOPA when the pump wavelength is set at $1555 \mathrm{~nm}$.

Fig. 9 shows the autocorrelation traces of different signal wavelengths measured by the autocorrelator. The real full-width half-maximum (FWHM) pulsewidth is calculated by assuming a $\operatorname{sech}^{2}$ pulse shape, multiplied the FWHM correlation width 

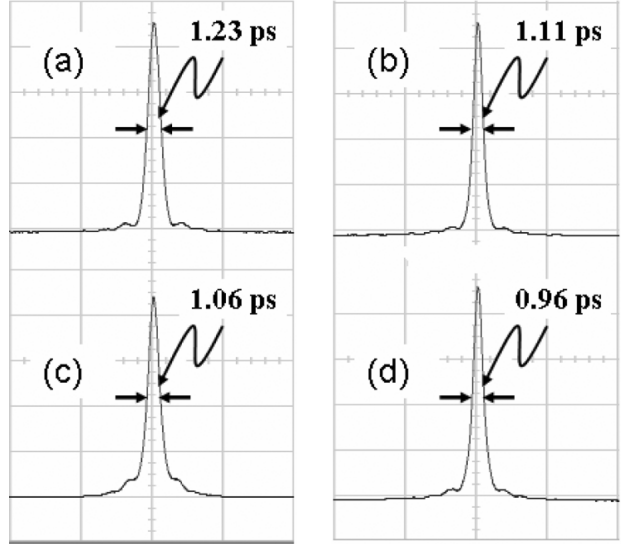

Fig. 9. Signal autocorrelation traces at different wavelengths. The signal wavelength in nanometers are : (a) 1610; (b) 1600; (c) 1590; (d) 1584.

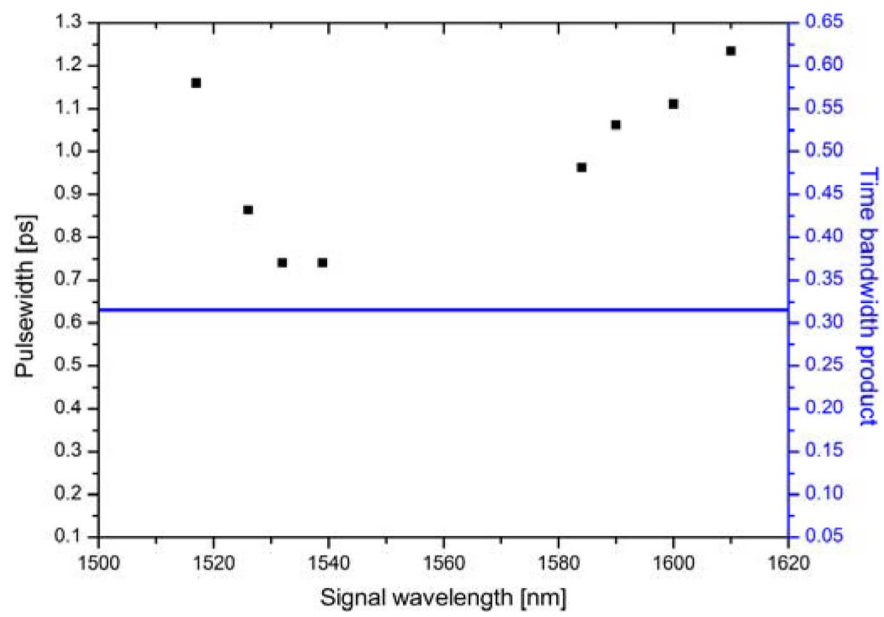

Fig. 10. Pulsewidth and time-bandwidth product versus signal wavelength. The horizontal line shows the time-bandwidth product of the transform-limited pulse.

using a deconvolution factor of 0.648 [26]. The spectral width of signal at all wavelengths is $4 \mathrm{~nm}$, same as the bandwidth of the VBTBPF.

Fig. 10 shows the pulsewidth and the time-bandwidth product (TBP) as a function of signal wavelength. It can be observed that the pulsewidth of the idler is narrower than that of the pump (3.5 ps) because of the pulse compression effect [4]. The compression ratio matched well with the theoretical value of 4 to 5 [4] when the signal is close to the pump thus the walk-off between the signal and the pump is small. However, when the signal is detuned far away from the pump, the walk-off becomes larger, thus makes the signal pulse broader and the compression ratio smaller (2.5 to 4$)$. The TBP is calculated to be around 0.37 to 0.61 , which is slightly larger than that of the transform-limited soliton pulse, 0.315 . The horizontal line in Fig. 10 shows the time-bandwidth product of the transformlimted pulse. This indicates that nearly-transform-limited pulses are generated across a $60-\mathrm{nm}$ tuning range. This tuning range of nearly-transform-limited pulse around $1550 \mathrm{~nm}$, is larger than those picosecond FOPOs reported [16]-[19].

The main limiting factor of extending the tuning range of the sub-picosecond pulse generator is the walk-off between the pump and the signal. The larger the frequency detune, the larger the walk-off between the pump and signal, thus leading to a shorter interaction length of the signal and pump and a lower parametric gain. For a 50-nm wavelength separation between the pump and the signal, the walk-off will be 2 ps approximately for a 50-m HNL-DSF with a dispersion slope of $0.035 \mathrm{ps} / \mathrm{nm}^{2} / \mathrm{km}$ by using (6) and (7). If we can use a relatively shorter fiber, for example, a fiber length of $10 \mathrm{~m}$, a 2-ps walk-off will corresponding to a 112-nm separation, thus the wavelength tunability can be improved. That suggests such kind of FOPO has a potentially wide tunability.

\section{CONCLUSION}

In this paper, a scheme to generate picosecond pulse based on narrowband OPA is demonstrated. By launching high power pulsed-pump in C-band into a short piece of HNL-DSF, parametric gain is observed at both S-band and L-band. With picosecond pulsed pump, high quality pulse is generated at 1460-nm with pulsewidth narrower than that of the pump. A widely-tunable picosecond FOPO based on HNL-DSF is also demonstrated. The output is continuously tunable from $1511 \mathrm{~nm}$ to $1541 \mathrm{~nm}$ and from $1583 \mathrm{~nm}$ to $1613 \mathrm{~nm}$ when the pump wavelength is fixed at $1555 \mathrm{~nm}$. Nearly-transform-limited sub-picosecond pulses are generated with compression factor of 2.5 to 5 . All these techniques would be useful for generating short pulse in non-conventional wavelength bands.

\section{ACKNOWLEDGMENT}

The authors would like to acknowledge Sumitomo Electric Industries for providing the HNL-DSF and Alnair Laboratories for providing the VBTBPF.

\section{REFERENCES}

[1] M. E. Marhic, Fiber Optical Parametric Amplifiers, Oscillators and Related Devices. Cambridge, U.K.: Cambridge Univ. Press, 2007.

[2] M. E. Marhic, K. K.-Y. Wong, and L. G. Kazovsky, "Wide-band tuning of the gain spectra of one-pump fiber optical parametric amplifiers," IEEE J. Sel. Topics Quantum Electron., vol. 10, no. 5, pp. 1133-1141, Sep./Oct. 2004.

[3] T. Torounidis, P. A. Andrekson, and B.-E. Olsson, "Fiber-optical parametric amplifier with 70-dB gain," IEEE Photon. Technol. Lett., vol. 18, no. 10, pp. 1194-1196, May 2006.

[4] T. Torounidis, M. Karlsson, and P. A. Andrekson, "Fiber optical parametric amplifier pulse source: Theory and experiments," J. Lightw. Technol., vol. 23, no. 12, pp. 4067-4073, Dec. 2005.

[5] B. P. P. Kuo, K. K. Y. Cheung, Y. Zhou, and K. K. Y. Wong, "Wideband generation of picosecond pulse via idler generation in optical parametric amplifier," presented at the 2009 IEEE/LEOS Winter Topical Meetings, Innsbruck, Austria, paper TuC3.5.

[6] M. H. Dunn and M. Ebrahimzadeh, "Parametric generation of tunable light from continuous-wave to femtosecond pulses," Science, vol. 286, pp. 1513-1517, Nov. 19, 1999

[7] J. E. Sharping, "Microstructure fiber based optical parametric oscillators," J. Lightw. Technol., vol. 26, no. 14, pp. 2184-2191, 2008.

[8] M. E. Marhic, K. K. Y. Wong, L. G. Kazovsky, and T. E. Tsai, "Continuous-wave fiber optical parametric oscillator," Opt. Lett., vol. 27, no. 16, pp. 1439-1441, 2002.

[9] C. De Matos, J. Taylor, and K. Hansen, "Continuous-wave, totally fiber integrated optical parametric oscillator using holey fiber," Opt. Lett. vol. 29, no. 9, pp. 983-985, 2004.

[10] T. Torounidis and P. Andrekson, "Broadband single-pumped fiber-optic parametric amplifiers," IEEE Photon. Technol. Lett., vol. 19 , no. 9, pp. 650-652, 2007.

[11] G. K. L. Wong, S. G. Murdoch, R. Leonhardt, J. D. Harvey, and V. Marie, "High-conversion-efficiency widely-tunable all-fiber optical parametric oscillator," Opt. Expr., vol. 15, no. 6, pp. 2947-2952, 2007. 
[12] Y. Deng, Q. Lin, F. Lu, G. Agrawal, and W. Knox, "Broadly tunable femtosecond parametric oscillator using a photonic crystal fiber," Opt. Lett., vol. 30, no. 10, pp. 1234-1236, 2005.

[13] Y. Q. Xu, S. G. Murdoch, R. Leonhardt, and J. D. Harvey, "Widely tunable photonic crystal fiber Fabry-Perot optical parametic oscillator," Opt. Lett., vol. 33, no. 12, pp. 1351-1353, 2008.

[14] J. E. Sharping, M. Fiorentino, P. Kumar, and R. S. Windeler, "Optical-parametric oscillator based on four-wave mixing in microstructure fiber," Opt. Lett., vol. 27, no. 19, pp. 1675-1677, 2002.

[15] J. E. Sharping, M. A. Foster, A. L. Gaeta, J. Lasri, O. Lyngnes, and $\mathrm{K}$. Vogel, "Octave-spanning, high-power microstrcture-fiber-based optical parametric oscillators," Opt. Expr., vol. 15, no. 4, pp. 1474-1479, 2007.

[16] J. Lasri, P. Devgan, R. Tang, J. E. Sharping, and P. Kumar, "A microstructure-fiber-based $10-\mathrm{GHz}$ synchronized tunable optical parametric oscillator in the 1550-nm regime," IEEE Photon. Technol. Lett., vol. 15, no. 8, pp. 1058-1060, 2003.

[17] D. K. Serkland and P. Kumar, "Tunable fiber-optic parametric oscillator," Opt. Lett., vol. 24, no. 2, pp. 92-94, 1999.

[18] P. Devgan, J. Lasri, R. Tang, V. Grigoryan, W. Kath, and P. Kumar, "10-GHz dispersion-managed soliton fiber-optical parametric oscillator using regenerative mode locking," Opt. Lett., vol. 30, no. 5, pp. 528-530, 2005.

[19] B. Cuenot, A. D. Ellis, and C. J. McKinstrie, "Widely-tunable modelocked multiwavelength ring laser," in Proc. ECOC, 2006, paper We3.P.19.

[20] Y. Zhou, K. K. Y. Cheung, S. Yang, P. C. Chui, and K. K. Y. Wong, "Widely-tunable picosecond optical parametric oscillator using highlynonlinear fiber," Opt. Lett., vol. 34, no. 7, pp. 989-991, 2009.

[21] Y. Zhou, K. K. Y. Cheung, S. Yang, P. C. Chui, and K. K. Y. Wong, "A time-dispersion tuned picosecond fiber optical parametric oscillator," IEEE Photon. Technol. Lett., vol. 21, no. 17, pp. 1223-1225, 2009.

[22] M. Karlson, "Four-wave mixing in fibers with randomly varying zerodispersion wavelength," J. Opt. Soc. Am. B, vol. 15, pp. 2269-2275, 1998.

[23] M. Farahmand and M. De Sterke, "Parametric amplification in presence of dispersion fluctuations," Opt. Expr., vol. 12, pp. 136-142, 2004.

[24] J. M. C. Boggio, J. R. Windmiller, M. Knutzen, R. Jiang, C. Bres, N. Alic, B. Stossel, K. Rottwitt, and S. Radic, "730-nm optical parametric conversion from near- to short-wave infrared band," Opt. Expr., vol. 16 , pp. 5435-5443, 2008.

[25] G. P. Agrawal, Nonlinear Fiber Optics, 3rd ed. San Diego, CA: Academic, 2001.

[26] Autocorrelator Instruction Manual, Femtochrome Research, Inc., FR-103MN.

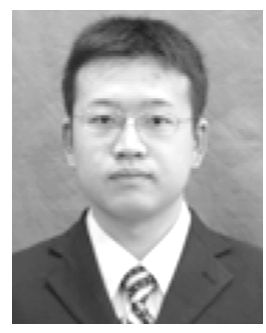

Yue Zhou received the B.Eng. degree in electronic engineering from Shanghai Jiao Tong University, China, in 2008 . He is currently working towards the M.Phil. degree in the Department of Electrical and Electronic Engineering, University of Hong Kong. His current research interests are fiber optical parametric amplifiers and oscillators.

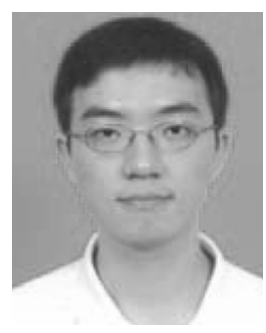

Bill Ping-Piu Kuo received the B.Eng. degree with first-class honors in electronic and communications engineering from the University of Hong Kong in 2006, and the M.Phil. degree in electronic engineering from the University of Hong Kong in 2008. $\mathrm{He}$ is currently working towards the Ph.D. degree in electronic engineering at the University of California at San Diego.

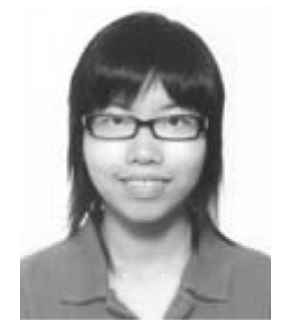

Kim K. Y. Cheung received the B.Eng. degree in electronic and communications engineering from the University of Hong Kong in 2008. She is currently working towards the M.Phil. degree in electrical and electronic engineering at the University of Hong Kong. Her current research interests are fiber optical parametric amplifiers and oscillators.

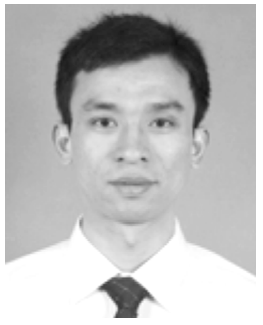

Sigang Yang received the B.E. degree in material science from Northeastern University, Shenyang, China, in 2000, the M.S. degree in physical electronics from Huazhong University of Science and Technology, Wuhan, China, in 2003. He received the Ph.D. degree in electronic engineering from Tsinghua University, Beijing, China, in 2008.

$\mathrm{He}$ is currently with the Photonic Systems Research Laboratory, Department of Electrical and Electronic Engineering, University of Hong Kong. His past research interests includes photonic crystal fibers and slow light. His current research interests include fiber optical parametric amplification and oscillators.

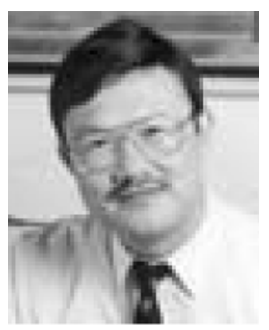

P. C. Chui received the M.Sc. degree from California State University.

$\mathrm{He}$ is currently a Lecturer in the Department of Electrical and Electronic Engineering, University of Hong Kong.

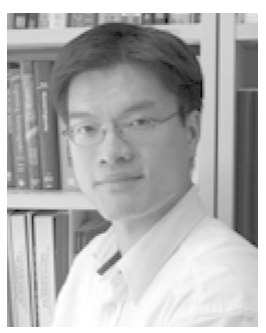

Kenneth Kin-Yip Wong (S'00-M'03) received the combined B.E. (1st class honors with medal award) degree in electrical engineering and B.S. degree in physics from the University of Queensland, Brisbane, Australia, in 1997, and the M.S. and Ph.D. degrees from Stanford University, Stanford, CA, in 1998 and 2003, respectively, both in electrical engineering.

He was a member of the Photonics and Networking Research Laboratory at Stanford University. During 1998-1999, he was with Hewlett-Packard Laboratories as a Research Engineer and contributed in projects that included parallel optics and vertical-cavity surface-emitting laser (VCSEL). He also worked as an Independent Consultant with Innovation CORE (a Sumitomo Electric Company), CA, in 2004. He is currently an Assistant Professor in the Department of Electrical and Electronic Engineering, University of Hong Kong, Hong Kong. He is the author or coauthor of more than 50 journal and conference papers. His past research interests include dense wavelength-division multiplexing (DWDM) systems, subcarrier multiplexed (SCM) optical systems, fiber nonlinearity, fiber optical parametric amplifiers, and photonic crystal fibers.

Dr. Wong is a member of The International Society for Optical Engineers (SPIE) and the IEEE Lasers and Electro-Optic Society (LEOS). He was the recipient of the Optical Society of America (OSA) New Focus Student Award and the IEEE/Lasers and Electro-Optics (LEOS) Graduate Student Fellowship, both in 2003. He was also the recipient of the Best Teacher Award in 2005-2006 from the University of Hong Kong. He is the Reviewer for Optics Letters, Journal of the Optical Society of America B, Optical Physics, Optics Express, IEEE PHOtONICS TECHNOLOGY LETTERS, IEEE/OSA JOURNAL OF LIGHTWAVE TECHNOLOGY, IEE Electronics Letters, and Optics Communications. 